Research article

\title{
Treatment of established postoperative nausea and vomiting: a quantitative systematic review
}

\author{
Faranak Kazemi-Kjellberg , Iris Henzi and Martin R Tramèr *
}

Address: Division of Anaesthesiology, Department Anaesthesiology, Clinical Pharmacology \& Surgical Intensive Care, Geneva University Hospitals, Geneva, Switzerland

E-mail: Faranak Kazemi-Kjellberg - faranak.k@spray.se; Iris Henzi - iris.henzi@hcuge.ch; Martin R Tramèr* - martin.tramer@hcuge.ch ${ }^{*}$ Corresponding author

Published: 26 October 200I

BMC Anesthesiology 200I, I:2
Received: 29 August 2001

Accepted: 26 October 2001

This article is available from: http://www.biomedcentral.com/I47I-2253/I/2

(c) 200I Kazemi-Kjellberg et al; licensee BioMed Central Ltd. Verbatim copying and redistribution of this article are permitted in any medium for any non-commercial purpose, provided this notice is preserved along with the article's original URL. For commercial use, contact info@biomedcentral.com

\begin{abstract}
Background: The relative efficacy of antiemetics for the treatment of postoperative nausea and vomiting (PONV) is poorly understood.

Methods: Systematic search (MEDLINE, Embase, Cochrane Library, bibliographies, any language, to 8.2000) for randomised comparisons of antiemetics with any comparator for the treatment of established PONV. Dichotomous data on prevention of further nausea and vomiting, and on side effects were combined using a fixed effect model.
\end{abstract}

Results: In seven trials (I,267 patients), I I different antiemetics were tested without placebos; these data were not further analysed. Eighteen trials $(3,809)$ had placebo controls. Dolasetron 12.5-100 mg, granisetron $0.1-3 \mathrm{mg}$, tropisetron $0.5-5 \mathrm{mg}$, and ondansetron $\mathrm{I}-8 \mathrm{mg}$ prevented further vomiting with little evidence of dose-responsiveness; with all regimens, absolute risk reductions compared with placebo were $20 \%-30 \%$. The anti-nausea effect was less pronounced. Headache was dose-dependent. Results on propofol were contradictory. The NK I antagonist GR205I7I, isopropyl alcohol vapor, metoclopramide, domperidone, and midazolam were tested in one trial each with a limited number of patients.

Conclusions: Of 100 vomiting surgical patients receiving a $5-\mathrm{HT}_{3}$ receptor antagonist, 20 to 30 will stop vomiting who would not have done so had they received a placebo; less will profit from the anti-nausea effect. There is a lack of evidence for a clinically relevant dose-response; minimal effective doses may be used. There is a discrepancy between the plethora of trials on prevention of PONV and the paucity of trials on treatment of established symptoms. Valid data on the therapeutic efficacy of classic antiemetics, which have been used for decades, are needed.

\section{Introduction}

Postoperative nausea and vomiting (PONV) are among the most common adverse events after surgery and anaesthesia. Compared with other postoperative complications (for instance, wound infection, deep vein thrombosis or myocardial ischemia), $\mathrm{PONV}$ is of minor medical importance; it almost never kills, and it never becomes chronic. However, PONV may be very distressing for patients. Workload for nursing staff dealing with vomiting patients is increased. In ambulatory surgery, intractable PONV may lead to unanticipated hospital admission. 
In France, 10\% percent of the population underwent an anaesthetic procedure in 1996 [1]. On average $30 \%$ of patients are suffering from PONV symptoms [2], and $1 \%$ of surgical outpatients need to be admitted to hospital due to intractable PONV [3-5]. Extrapolating these numbers to the UK suggests that every year almost 2'0oo'ooo people suffer from PONV symptoms, and about 20'0oo outpatients need to be admitted following ambulatory surgery due to intractable PONV. Thus, PONV is likely to create considerable extra costs for health care systems.

Much research on the control of PONV has been conducted during the last four decades. The majority of clinical trials focuses on prophylaxis of PONV (i.e. patients receive an antiemetic at induction of anaesthesia, during surgery or shortly before they wake up). There are, however, several problems with the prevention of PONV. First, the efficacy of prophylactic antiemetic interventions in the daily surgical setting (i.e. when the baseline risk for PONV is not particularly high) is often disappointing [2]. Second, there is no evidence that prophylaxis decreases the likelihood of unanticipated admission [2]. Third, prophylaxis of PONV is likely to be less costeffective than treatment of established symptoms [6]. And finally, with prevention strategies, patients who actually do not need any prophylaxis are unnecessarily exposed to a drug, and are thus put at risk of suffering from unnecessary adverse drug reactions.

Efficacious and safe treatment strategies for patients who are nauseated or who are vomiting after surgery are needed. The aim of this study was to systematically review the literature on valid data on any treatment of established PONV symptoms, to critically appraise the data, to test for dose-responsiveness for each drug, and to estimate relative efficacy and likelihood for harm of the various treatments.

\section{Methods}

\section{Systematic search}

We searched the MEDLINE (PubMed, from 1966), and EMBASE (from 1974) databases using different search strategies. We also searched the Cochrane Controlled Trials Register (Cochrane Library 2000, issue IV). We used the free text terms (postoperative OR postoperative $\mathrm{OR}$ postsurg *), (nausea $\mathrm{OR}$ vomiting $\mathrm{OR}$ emesis $\mathrm{OR}$ retching), (randomised OR randomized), (treatment), NOT (chemotherapy OR radiotherapy), NOT (prevention OR prophylaxis) and combinations of these terms. The date of the last electronic search was 21.8.2000. We checked reference lists of retrieved reports and relevant review articles, and we searched our own comprehensive in-house bibliography. Authors of original trials were contacted when there was ambiguity about the data. We did not contact manufacturers.

\section{Inclusion and exclusion criteria, validity assessment, data extraction}

We included full reports of randomised comparisons of any therapeutic antiemetic intervention (experimental intervention) with placebo, no treatment or another antiemetic (control intervention) in vomiting or nauseated postoperative patients. When Intralipid ${ }^{\circledR}$ was used as a control in propofol trials (to maintain blinding due to its milky-white colour), it was considered as an inactive control.

Retrieved reports were screened by one author (FK). Reports, which did not clearly meet inclusion criteria were excluded at this stage. All potentially relevant reports were then read by all authors independently who scored them for methodological validity using the three-item, five point Oxford scale taking into account randomisation, double-blinding, and description of withdrawals [7]. The minimum validity requirement for an included trial was an adequate method of randomisation (for instance, a table of random numbers). Trials with pseudorandomisation (for instance, according to patients' date of birth) were excluded.

The main endpoint of efficacy was a "success" (i.e. no further nausea or vomiting in a nauseated or vomiting patient). According to previous analyses [8], and in agreement with the majority of all retrieved trials, we distinguished between two arbitrarily defined observations periods: "early success" was within or close to 6 hours after administration of the study drugs, and "late success" was within or close to 24 hours. Dichotomous data on anti-vomiting and anti-nausea efficacy were separately extracted, and separately analysed. When no distinction was made between nausea and vomiting, the data were not further analysed. Data on adverse drug reactions were analysed when they were reported in dichotomous form. Data on patients' satisfaction, duration of hospital stay, number of vomiting episodes, degree of nausea, or number of rescue treatments were not analysed since these data were inconsistently reported. Sponsorship was assumed when it was acknowledged in the original paper or when a representative of the manufacturer was a co-author of the paper. All data were extracted by one author (FK) and then checked by the two others independently. Authors met to agree consensus on validity scores and extracted data; discrepancies were resolved by discussion.

\section{Analyses}

For both efficacy and harm we calculated relative risks with $95 \%$ confidence intervals [9]. A statistically significant difference between an experimental intervention and control was assumed when the 95\% confidence interval around the relative risk did not include 1. Data 
from independent trials were combined only when the data represented clinically homogenous subgroups. Such subgroups would include comparisons of data of the same dose and route of administration of the same experimental intervention, with the same control intervention (for instance, a placebo), and reporting on the same emesis endpoint (for instance vomiting) during the same observation period (for instance late success). A fixed effect model was used to combine these clinically homogenous data [10].

It became clear that valid trials represented homogenous patient populations with minimal variations in baseline risks and outcome frequencies. Also, most antiemetic interventions were tested in one or two large multicentre studies with similar control event rates. As estimates of the clinical relevance of the antiemetic efficacy of the treatments, we, therefore, calculated absolute risk reductions compared with placebo. We also calculated the reciprocal of the absolute risk reduction, the numberneeded-to-treat [11], with 95\% confidence interval [12].

Dose-responsiveness was tested for as with previous similar analyses $[8,13]$, taking into account criteria of both statistical significance and clinical relevance of differences in efficacy between doses. Since control event rates were very similar in these trials, we were using the absolute risk reduction (and the number-needed-totreat) to test for dose-responsiveness. For homogeneous subgroups, absolute risk reductions (numbers-neededto-treat) were graphically plotted as recently suggested [14]. A consistent increase in the absolute risk reduction (a decrease in the number-needed-to-treat) with increasing doses was interpreted as weak evidence of dose-responsiveness. A statistically significant difference between two doses, and thus strong evidence of dose-responsiveness, was assumed when the $95 \%$ confidence intervals of the absolute risk reductions (numbers-neededto-treat) of these two doses did not overlap, or when the higher dose was significantly different from control and the lower dose was not. According to a pre-hoc decision in the clinical setting of PONV [15], a change of the absolute risk reduction by $\bullet 20 \%$ was regarded as a clinically relevant degree of change in antiemetic efficacy. For instance, a decrease of the number-needed-to-treat from 5 to 4 compared with placebo to prevent further vomiting in a vomiting patient by increasing the dose would be regarded as a worthwhile improvement. This would then justify an increase of the dose $[8,13]$.

\section{Results}

\section{Included and excluded articles}

We screened 55 reports; 34 of those were potentially relevant for the purpose of this study (Figure 1). Nine had to be subsequently excluded. Data of one large sponsored

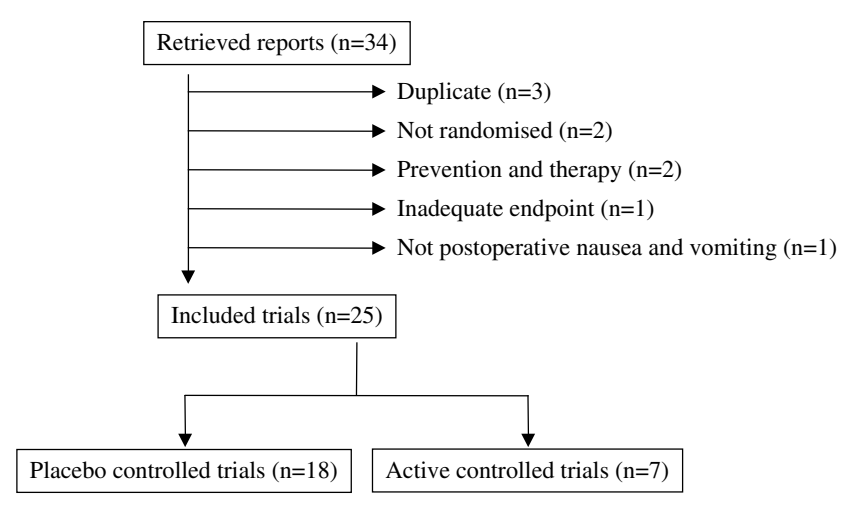

Figure I

Flowchart of retrieved and analysed reports.

multicentre trial on ondansetron (500 patients) [16] were published in two further full reports $[17,18]$, and data of one large sponsored multicentre trial on dolasetron (620 patients) [19] were published in one subsequent full report [20]; the original reports only were considered by us $[16,19]$. In two trials, treatment allocation was not randomised; one tested ondansetron [21], the other pepermint oil [22]. In two trials, patients received both prophylactic and therapeutic antiemetic interventions, and efficacy data could not been separated; one trial tested droperidol and metoclopramide [23], the other droperidol, metoclopramide, and domperidone [24]. One trial did not report on efficacy data which were relevant for the purpose of this analysis [25]. Finally, one report was on patients with nausea and vomiting due to oesophageal and gastric disorders [26].

Twenty-five trials fulfilled our inclusion criteria. One on domperidone was published in 1980 [27], all others were published after 1990; 11 trials (44\%) were published since 1997. One report was in German [28] all others were in English. Three trials were in children [29-31], all others were in adults. In the 17 trials, which reported on the number of patients who were followed up before randomisation, 12,107 patients were followed up and 3,572 of those vomited or felt nauseous before treatment (average $30 \%$, range $9 \%$ [32] to $63 \%$ [33]). We contacted the main authors of two trials to clarify inconsistencies in the reporting of late success data $[34,35]$. One author only responded to our enquiry [34]; these data were included in our analyses.

\section{Active controlled trials}

In seven randomised trials $(1,267$ patients $)$ [28,29,32,36-39], eleven different experimental interventions were tested without a placebo group (Table 1). One antiemetic intervention was tested in three trials, five in two each, and five in one each. Because of the large 
Table I: Active-controlled trials

\begin{tabular}{|c|c|c|c|c|}
\hline Ref & $\begin{array}{l}\text { Validity score } \\
\text { random blinding dropouts }\end{array}$ & $\begin{array}{c}\text { Comparison } \\
\text { (number of patients in brackets) }\end{array}$ & Surgery & Sponsorship \\
\hline \multirow[t]{2}{*}{ [28] } & $1 / 1 / 0$ & I) Ondansetron 8 mg iv (50) & Not reported & \\
\hline & & 2) Droperidol I. 25 mg iv (50) & & \\
\hline \multirow[t]{2}{*}{ [29] } & $2 / 2 / 1$ & I) Ondansetron $0.1 \mathrm{mg} / \mathrm{kg}$ iv (16) & Extraabdominal & \\
\hline & & 2) Droperidol $20 \mu \mathrm{g} / \mathrm{kg}$ iv (I3) & & \\
\hline \multirow[t]{3}{*}{ [32] } & $2 / 2 / 0$ & I) Droperidol I.25 mg iv (27) & Mixed & \\
\hline & & 2) Metoclopramide $10 \mathrm{mg}$ iv (25) & & \\
\hline & & 3) Propofol $10 \mathrm{mg}$ iv (26) & & \\
\hline \multirow[t]{2}{*}{ [36] } & $1 / 1 / 0$ & I) Ondansetron 4 mg iv (380) & Not reported & Glaxo \\
\hline & & 2) Metoclopramide $10 \mathrm{mg}$ iv (366) & & \\
\hline \multirow[t]{3}{*}{ [37] } & $1 / 1 / 0$ & I) Droperidol $20 \mu \mathrm{g} / \mathrm{kg}$ iv (40) & Laparoscopic & \\
\hline & & 2) Granisetron $40 \mu \mathrm{g} / \mathrm{kg}$ iv (40) & cholecystectomy & \\
\hline & & 3) Metoclopramide $0.2 \mathrm{mg} / \mathrm{kg}$ iv (40) & & \\
\hline \multirow[t]{3}{*}{ [38] } & $1 / 1 / 0$ & I) Droperidol $20 \mu \mathrm{g} / \mathrm{kg}$ iv (40) & Major & \\
\hline & & 2) Granisetron $40 \mu \mathrm{g} / \mathrm{kg}$ iv (40) & gynecological & \\
\hline & & 3) Metoclopramide $0.2 \mathrm{mg} / \mathrm{kg}$ iv (40) & & \\
\hline \multirow[t]{3}{*}{ [39] } & $1 / 1 / 1$ & I) Alizapride 100 mg iv (24) & Gynecological & Glaxo \\
\hline & & 2) Droperidol I mg iv (25) & & \\
\hline & & 3) Ondansetron 8 mg iv (25) & & \\
\hline
\end{tabular}

variety in different experimental interventions and the inconsistency in control interventions, these data were not further analysed.

\section{Placebo controlled trials}

In 18 trials ( 3,809 patients), there was an inactive control group [16,17,19,27,30,31,33-35,40-48] (Table 2). Most trials reported separately on anti-nausea and antivomiting data. Three trials also reported on combined nausea and vomiting data [33-35], and two (one tested ondansetron and metoclopramide [45], and one tested domperidone [27]) reported only on combined data. Combined data were not further analysed.

Eleven trials (61\% of all placebo-controlled trials) tested four $5-\mathrm{HT}_{3}$ receptor antagonists (dolasetron, granisetron, ondansetron, or tropisetron) in 3,427 patients (90\% of all patients in placebo-controlled trials). Average trial size was 312 patients (range, 36 to 620). Median validity score was 3 (range, 2 to 5). Most data came from multicentre dose-finding studies that were sponsored by the manufacturers of the respective $5-\mathrm{HT}_{3}$ receptor antagonists.

Three trials tested propofol $[35,46,47]$, and one each midazolam [48], a $\mathrm{NK}_{1}$ antagonist (GR205171) [33], domperidone [27], and isopropyl alcohol vapor [31] in 382 patients. In those trials, average size was 55 patients (range, 20 to 100). This was significantly different from the trials on $5-\mathrm{HT}_{3}$ receptor antagonists $(\mathrm{p}=0.002)$. Me- dian validity score was 2 (range, 2 to 4 ). This was not different from trials on $5-\mathrm{HT}_{3}$ receptor antagonists $(\mathrm{p}=$ o.126). Two trials were sponsored [33,46].

\section{5- $\mathrm{HT}_{3}$ receptor antagonists}

Granisetron $0.1 \mathrm{mg}, 1 \mathrm{mg}$, and $3 \mathrm{mg}$ (386 patients received granisetron) and tropisetron $0.5 \mathrm{mg}, 2 \mathrm{mg}$, and 5 $\mathrm{mg}$ (237) were tested in one multicentre trial each [34,41]. Dolasetron 12.5, $25 \mathrm{mg}, 50 \mathrm{mg}$, and $100 \mathrm{mg}$ was tested in two multicentre trials $(765)[19,40]$. Data on ondansetron came from seven trials. Three doses, $1 \mathrm{mg}$, $4 \mathrm{mg}$, and $8 \mathrm{mg}$ were tested in 839 adults in two multicentre $[16,17]$ and four single centre trials $[42-45]$, and $0.1 \mathrm{mg}$ and $16 \mathrm{mg}$ were tested in 60 adults in one trial [43]. Finally, $0.1 \mathrm{mg} / \mathrm{kg}$ was tested in 192 children in one trial [30]; this was the only paediatric trial that tested a setron.

Graphical display suggested similar anti-vomiting efficacy with all $5-\mathrm{HT}_{3}$ receptor antagonists, and little evidence of dose-responsiveness (Table 3, Figure 2). For early success, relative risk point estimates were between 1.6 and 2.2, and numbers-needed-to-treat point estimates were between 3.0 and 4.7. According to our predefined criteria, there was some evidence of dose-responsiveness with ondansetron; the lowest dose tested, $0.1 \mathrm{mg}$, was no different from placebo, and all higher doses were significantly more efficacious than placebo. With the main fixed doses (1 mg, $4 \mathrm{mg}$, and $8 \mathrm{mg}$ ), however, there was no clinically relevant dose-response; the number-need- 
Table 2: Placebo-controlled trials

\begin{tabular}{|c|c|c|c|c|c|c|c|c|c|c|}
\hline \multirow[t]{3}{*}{ Ref } & \multirow{3}{*}{$\begin{array}{l}\text { Validity } \\
\text { score } \\
\text { random } \\
\text { blinding } \\
\text { dropouts }\end{array}$} & \multirow{3}{*}{$\begin{array}{l}\text { Comparison } \\
\qquad \begin{array}{c}\text { (number of patients in } \\
\text { brackets) }\end{array}\end{array}$} & \multirow[t]{3}{*}{ Surgery } & \multicolumn{3}{|c|}{ Early success } & \multicolumn{3}{|c|}{ Late success } & \multirow{3}{*}{ Sponsorship } \\
\hline & & & & \multirow[b]{2}{*}{ time point } & \multicolumn{2}{|c|}{ absence of } & \multicolumn{3}{|c|}{ absence of } & \\
\hline & & & & & nausea & vomiting & time point & nausea & vomiting & \\
\hline \multicolumn{11}{|c|}{ Dolasetron } \\
\hline$[40]$ & $\mathrm{I} / \mathrm{I} / 0$ & $\begin{array}{l}\text { 1) Dolasetron } 12.5 \mathrm{mg} \text { iv }(66) \\
\text { 2) Dolasetron } 25 \mathrm{mg} \text { iv }(65) \\
\text { 3) Dolasetron } 50 \mathrm{mg} \text { iv }(67) \\
\text { 4) Dolasetron } 100 \mathrm{mg} \text { iv }(68) \\
\text { 5) Placebo iv (7I) }\end{array}$ & Mixed & & no data & & $24 \mathrm{~h}$ & no data & $\begin{array}{l}16 / 66 \\
18 / 65 \\
25 / 67 \\
17 / 68 \\
8 / 71\end{array}$ & $\begin{array}{l}\text { Hoechst } \\
\text { Marion } \\
\text { Russel }\end{array}$ \\
\hline$[19]$ & $2 / 1 / 0$ & $\begin{array}{l}\text { I) Dolasetron } 12.5 \mathrm{mg} \text { iv }(130) \\
\text { 2) Dolasetron } 25 \mathrm{mg} \text { iv (II9) } \\
\text { 3) Dolasetron } 50 \mathrm{mg} \text { iv }(124) \\
\text { 4) Dolasetron } 100 \mathrm{mg} \text { iv (I26) } \\
\text { 5) Placebo iv (I2I) }\end{array}$ & Mixed & $2 \mathrm{~h}$ & no data & $\begin{array}{l}72 / 130 \\
60 / 119 \\
60 / 124 \\
64 / 126 \\
33 / 121\end{array}$ & $24 \mathrm{~h}$ & no data & $\begin{array}{l}46 / 130 \\
33 / 119 \\
36 / 124 \\
37 / 126 \\
13 / 121\end{array}$ & $\begin{array}{l}\text { Hoechst } \\
\text { Marion } \\
\text { Russel }\end{array}$ \\
\hline \multicolumn{11}{|c|}{ Granisetron } \\
\hline$[41]$ & $2 / 1 / 1$ & $\begin{array}{l}\text { I) Granisetron } 0.1 \mathrm{mg} \text { iv (I28) } \\
\text { 2) Granisetron I mg iv (I33) } \\
\text { 3) Granisetron } 3 \mathrm{mg} \text { iv (I25) } \\
\text { 4) Placebo iv (I33) }\end{array}$ & Mixed & $6 \mathrm{~h}$ & $\begin{array}{l}51 / 128 \\
54 / 133 \\
53 / 125 \\
22 / 133\end{array}$ & $\begin{array}{l}68 / 128 \\
77 / 133 \\
75 / 125 \\
35 / 133\end{array}$ & $24 \mathrm{~h}$ & $\begin{array}{l}34 / 128 \\
40 / 133 \\
46 / 125 \\
17 / 133\end{array}$ & $\begin{array}{l}49 / 128 \\
61 / 133 \\
61 / 125 \\
26 / 133\end{array}$ & \\
\hline \multicolumn{11}{|c|}{ Tropisetron } \\
\hline [34] & $1 / 2 / 0$ & $\begin{array}{l}\text { I) Tropisetron } 0.5 \mathrm{mg} \text { iv (77) } \\
\text { 2) Tropisetron } 2 \mathrm{mg} \text { iv }(80) \\
\text { 3) Tropisetron } 5 \mathrm{mg} \text { iv (80) } \\
\text { 4) Placebo iv (77) }\end{array}$ & Mixed & $4 \mathrm{~h}$ & $\begin{array}{l}45 / 77 \\
45 / 80 \\
45 / 80 \\
35 / 77\end{array}$ & $\begin{array}{l}54 / 77 \\
61 / 80 \\
59 / 80 \\
35 / 77\end{array}$ & $24 \mathrm{~h}$ & $\begin{array}{l}33 / 77 \\
36 / 80 \\
37 / 80 \\
26 / 77\end{array}$ & $\begin{array}{l}40 / 77 \\
46 / 80 \\
48 / 80 \\
22 / 77\end{array}$ & Sandoz \\
\hline \multicolumn{11}{|c|}{ Ondansetron } \\
\hline [42] & $2 / 2 / 0$ & $\begin{array}{l}\text { 1) Ondansetron } 8 \mathrm{mg} \text { iv (35) } \\
\text { 2) Placebo iv (36) }\end{array}$ & Laparoscopy & $2 \mathrm{~h}$ & no data & $\begin{array}{l}17 / 35 \\
3 / 36\end{array}$ & & no data & & Glaxo \\
\hline$[17]$ & $\mathrm{I} / \mathrm{I} / \mathrm{I}$ & $\begin{array}{l}\text { I) Ondansetron I mg iv (l }\left(\begin{array}{ll}I & 2\end{array}\right) \\
\text { 2) Ondansetron } 4 \mathrm{mg} \text { iv }\left(\begin{array}{ll}1 & \mathrm{I}\end{array}\right) \\
\text { 3) Ondansetron } 8 \mathrm{mg} \text { iv }\left(\begin{array}{ll}1 & 04\end{array}\right) \\
\text { 4) Placebo iv (I08) }\end{array}$ & $\begin{array}{c}\text { Not } \\
\text { specified }\end{array}$ & & no data & & $24 \mathrm{~h}$ & no data & $\begin{array}{l}45 / 112 \\
49 / 112 \\
43 / 104 \\
28 / 108\end{array}$ & Glaxo \\
\hline$[16]$ & $\mathrm{I} / \mathrm{I} / 0$ & $\begin{array}{l}\text { I) Ondansetron I mg iv (I30) } \\
\text { 2) Ondansetron } 4 \mathrm{mg} \text { iv (II9) } \\
\text { 3) Ondansetron } 8 \mathrm{mg} \text { iv (I22) } \\
\text { 4) Placebo iv (I29) }\end{array}$ & Mixed & $2 \mathrm{~h}$ & no data & $\begin{array}{l}74 / 130 \\
73 / 119 \\
70 / 122 \\
39 / 129\end{array}$ & $24 \mathrm{~h}$ & no data & $\begin{array}{l}53 / 130 \\
56 / 119 \\
57 / 122 \\
19 / 129\end{array}$ & Glaxo \\
\hline [30] & $2 / 2 / 1$ & $\begin{array}{l}\text { I) Ondansetron } 0.1 \mathrm{mg} / \mathrm{kg} \text { iv } \\
\text { (192) } \\
\text { 2) Placebo iv (183) }\end{array}$ & Paediatric & $2 \mathrm{~h}$ & no data & $\begin{array}{l}150 / 192 \\
63 / 183\end{array}$ & $24 \mathrm{~h}$ & no data & $\begin{array}{l}98 / 186 \\
30 / 179\end{array}$ & Glaxo \\
\hline$[44]$ & $2 / 1 / 0$ & $\begin{array}{l}\text { I) Ondansetron } 8 \mathrm{mg} \text { iv (18) } \\
\text { 2) Placebo iv (I8) }\end{array}$ & Mixed & $4 \mathrm{~h}$ & $\begin{array}{c}14 / 18 \\
5 / 18\end{array}$ & $\begin{array}{l}17 / 18 \\
17 / 18\end{array}$ & & no data & & Glaxo \\
\hline$[45]$ & $\mathrm{I} / \mathrm{I} / \mathrm{I}$ & $\begin{array}{l}\text { 1) Ondansetron } 4 \mathrm{mg} \text { iv (58) } \\
\text { 2) Metoclopramide } 10 \mathrm{mg} \text { iv } \\
\text { (57) } \\
\text { 3) Placebo iv (60) }\end{array}$ & $\begin{array}{l}\text { Gynaecologic } \\
\text { laparoscopy }\end{array}$ & $0.5 \mathrm{~h}$ & $\begin{array}{l}\text { Only cc } \\
\text { nauseat }\end{array}$ & $\begin{array}{l}\text { mbined } \\
\text { vomiting } \\
\text { ta }\end{array}$ & $48 \mathrm{~h}$ & $\begin{array}{r}\text { Only c } \\
\text { nausea } \\
d\end{array}$ & $\begin{array}{l}\text { mbined } \\
\text { vomiting } \\
\text { ta }\end{array}$ & \\
\hline
\end{tabular}


Table 2: Placebo-controlled trials (Continued)

\begin{tabular}{|c|c|c|c|c|c|c|c|c|c|c|}
\hline [43] & $2 / 2 / 0$ & $\begin{array}{l}\text { 1) Ondansetron } 0.1 \mathrm{mg} \text { iv (32) } \\
\text { 2) Ondansetron } 4 \mathrm{mg} \text { iv }(29) \\
\text { 3) Ondansetron } 16 \mathrm{mg} \text { iv (28) } \\
\text { 4) Placebo iv (32) }\end{array}$ & Mixed & $6 \mathrm{~h}$ & no data & $\begin{array}{l}7 / 32 \\
12 / 29 \\
15 / 28 \\
5 / 32\end{array}$ & $24 \mathrm{~h}$ & no data & $\begin{array}{l}5 / 32 \\
11 / 29 \\
14 / 28 \\
5 / 32\end{array}$ & Glaxo \\
\hline \multicolumn{11}{|c|}{ Propofol } \\
\hline [35] & $1 / 2 / 0$ & $\begin{array}{l}\text { 1) Propofol } 20 \mathrm{mg} \text { iv (24) } \\
\text { 2) Propofol } 40 \mathrm{mg} \text { iv (22) } \\
\text { 3) Intralipid iv (23) }\end{array}$ & Day case & $2 \mathrm{~h}$ & no data & $\begin{array}{l}21 / 24 \\
17 / 22 \\
13 / 23\end{array}$ & & no data & & \\
\hline [46] & $1 / 1 / 0$ & $\begin{array}{l}\text { 1) Propofol } 3 \mathrm{mg} \text { iv (12) } \\
\text { 2) Propofol } 9 \mathrm{mg} \text { iv (12) } \\
\text { 3) Propofol } 27 \mathrm{mg} \text { iv (12) } \\
\text { 4) Intralipid iv (12) }\end{array}$ & $\begin{array}{l}\text { Gynecologic } \\
\text { laparoscopy }\end{array}$ & $1.5 \mathrm{~h}$ & $\begin{array}{l}4 / 12 \\
3 / 12 \\
3 / 12 \\
3 / 12\end{array}$ & $\begin{array}{l}10 / 12 \\
11 / 12 \\
7 / 12 \\
10 / 12\end{array}$ & & no data & & $\begin{array}{l}\text { ICI Pharma- } \\
\text { ceuticals }\end{array}$ \\
\hline [47] & $2 / 1 / 0$ & $\begin{array}{l}\text { 1) Propofol } 0.2 \mathrm{mg} / \mathrm{kg} \text { iv (35) } \\
\text { 2) Intralipid (35) }\end{array}$ & $\begin{array}{l}\text { Adenectomy } \\
\text { Tonsillectomy }\end{array}$ & I h & no data & $\begin{array}{l}34 / 35 \\
30 / 35\end{array}$ & $24 \mathrm{~h}$ & no data & $\begin{array}{l}13 / 35 \\
20 / 35\end{array}$ & \\
\hline \multicolumn{11}{|c|}{ Others } \\
\hline [48] & $2 / 2 / 0$ & $\begin{array}{l}\text { I) Midazolam I mg iv + I mg/h } \\
\text { (10) } \\
\text { 2) Placebo iv (10) }\end{array}$ & Any surgery & $9 \mathrm{~h}$ & no data & $\begin{array}{l}9 / 10 \\
3 / 10\end{array}$ & & no data & & \\
\hline [33] & $1 / 1 / 0$ & $\begin{array}{l}\text { 1) GR205 I7I } 25 \mathrm{mg} \text { iv (I8) } \\
\text { 2) Placebo iv (I8) }\end{array}$ & Hysterectomy & $2 \mathrm{~h}$ & $\begin{array}{l}10 / 18 \\
4 / 18\end{array}$ & $\begin{array}{l}14 / 18 \\
5 / 18\end{array}$ & $24 \mathrm{~h}$ & \multicolumn{2}{|c|}{$\begin{array}{l}\text { Only combined } \\
\text { nausea+vomiting } \\
\text { data }\end{array}$} & Glaxo \\
\hline [27] & $1 / 1 / 0$ & $\begin{array}{l}\text { 1) Domperidone } 10 \mathrm{mg} \text { iv (52) } \\
\text { 2) Placebo iv (48) }\end{array}$ & Mixed & $6 \mathrm{~h}$ & \multicolumn{2}{|c|}{$\begin{array}{l}\text { Only combined } \\
\text { nausea+vomiting } \\
\text { data }\end{array}$} & & no data & & \\
\hline$[31]$ & $2 / 0 / 0$ & $\begin{array}{l}\text { 1) Isopropyl alcohol vapor } \\
\text { nasal }(20) \\
\text { 2) Saline nasal (19) }\end{array}$ & Paediatric & $\mathrm{Ih}$ & $\begin{array}{l}18 / 20 \\
8 / 19\end{array}$ & $\begin{array}{l}7 / 20 \\
2 / 19\end{array}$ & & no data & & \\
\hline
\end{tabular}

All trials are in adults, except "paediatric"

ed-to-treat for late anti-vomiting efficacy decreased from 4.8 to 4.1 (i.e. an improvement by $15 \%$ ). Best anti-vomiting efficacy was with granisetron, and there was some evidence of a clinically relevant dose-response. With the lowest dose tested ( $0.1 \mathrm{mg})$, the number-needed-to-treat for early success was 3.7. This number decreased to 3.2 (i.e. an improvement by $14 \%$ ) when the dose was increased by a factor of 10 (to $1 \mathrm{mg}$ ). When the dose was further increased by a factor of 30 (to $3 \mathrm{mg}$ ), the numberneeded-to-treat decreased to 3.0 (i.e. an improvement by $23 \%)$. For late success, the improvement was $11 \%$ and $36 \%$, when the dose was increased to $1 \mathrm{mg}$ and $3 \mathrm{mg}$, respectively. There was also some evidence of a clinically relevant dose-response for late anti-vomiting efficacy with tropisetron. With $0.5 \mathrm{mg}$, the number-needed-totreat to prevent further vomiting for 24 hours was 4.3 . When the dose was increased fourfold, the numberneeded-to-treat decreased to 3.5 (i.e. an improvement by $18 \%)$. When the dose was increased tenfold, the numberneeded-to-treat decreased to 3.2 (i.e. an improvement by 26\%).

Three trials only reported on anti-nausea efficacy; two multicentre trials on granisetron [41] and tropisetron [34], respectively, and one small trial on ondansetron [44] (Table 2). Anti-nausea efficacy was consistently less 


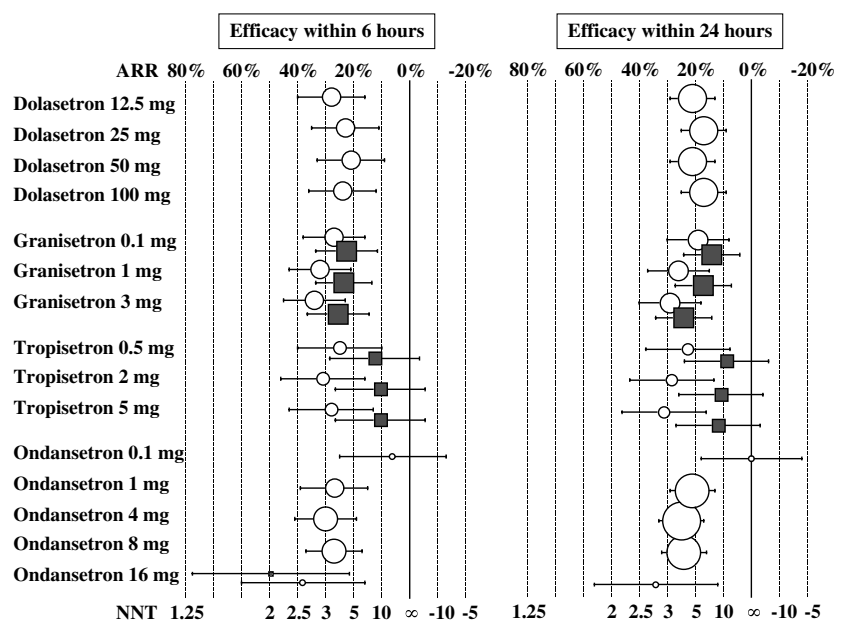

Figure 2

Prevention of further postoperative nausea and vomiting with $5-\mathrm{HT}_{3}$ receptor antagonists. Symbols are absolute risk reductions (ARR) or numbers-needed-to-treat (NNT), respectively, with $95 \%$ confidence intervals. Symbol sizes are proportional to the number of analysed patients. White circles: vomiting; grey squares: nausea. $\infty=$ infinity $(A R R=0)$. All data are from adults.

pronounced, compared with the anti-vomiting efficacy (Table 3, Figure 2). For tropisetron, none of the doses tested achieved a statistically significant anti-nausea effect, neither early nor late.

The most frequently reported adverse effect with $5-\mathrm{HT}_{3}$ receptor antagonists was headache (Table 4). There was some evidence of dose-responsiveness, although for none of the dose groups tested (i.e. low, medium or high) the result was statistically significant. With the lowest doses, the risk of headache was decreased compared with placebo. With the medium doses, there was equivalence. With the highest doses tested, the risk of headache was increased compared with placebo.

\section{Propofol}

Six different regimens of propofol were tested in three trials, each in a limited number of patients [35,46,47] (Table 2). Results were inconsistent. There was a lack of evidence of any dose-effect, or increased efficacy in particular groups of patients. When the data were combined, there was no evidence of a significant antivomiting effect (Table 3). One trial only tested early antinausea efficacy [46]; the effect was not statistically significant. In two trials, increased sedation with propofol was reported $[35,46]$. In one trial, one of 22 patients receiving propofol $40 \mathrm{mg}$ had an episode of apnoea [35].

\section{Others}

One trial each tested a $\mathrm{NK}_{1}$-receptor antagonist [33], midazolam [48], domperidone [27], nasal isopropyl alcohol [31], and metoclopramide [45] (Tables 2 and 3). Trials were on a limited number of patients, or reported combined nausea and vomiting success rates only.

None of the trials reported on the number of outpatients who needed to be admitted due to intractable PONV.

\section{Discussion}

There are three main results of this systematic review. First, $5-\mathrm{HT}_{3}$ receptor antagonists are efficacious to some extent in preventing further vomiting in a patient who is vomiting after surgery; they show less efficacy in preventing further nausea in a nauseated patient. Second, over wide ranges of doses there is weak evidence only of dose-responsiveness with these drugs. Third, although classic antiemetics (for instance, droperidol or metoclopramide) have been widely used for decades, there is a lack of evidence on their therapeutic efficacy in the postoperative period.

The $5-\mathrm{HT}_{3}$ receptor antagonists were the most frequently tested drugs. For all of them at least one large and well designed multicentre trial could be retrieved. Thus, for this class of antiemetics, treatment recommendations can be based on strong evidence. There were, however, some methodological problems with these trials. For instance, some of them were published more than once $[17,18,20]$. Also, there were no reports on direct comparisons of $5-\mathrm{HT}_{3}$ receptor antagonists. Our analyses had to rely on indirect comparisons from placebo-controlled trials. Finally, for most of these trials, reporting of efficacy data was incomplete. It is important to distinguish between anti-vomiting and anti-nausea efficacy since nausea is not a little vomiting [49]. An antiemetic may well stop further vomiting in a vomiting patient but leave the patient nauseous. For both granisetron and tropisetron, there was consistent evidence that the anti-nausea effect was less pronounced than the anti-vomiting effect. For the other setrons no nausea data were reported. This relative lack of anti-nausea efficacy of $5-\mathrm{HT}_{3}$ receptor antagonists has been well known from previous analyses on prophylactic ondansetron in the surgical setting [13], and on the antiemetic efficacy of $5-\mathrm{HT}_{3}$ receptor antagonists in patients undergoing radiotherapy [50]. The selective reporting of anti-vomiting efficacy data in the majority of these trials may lead to a biased perception of the antiemetic efficacy of $5-\mathrm{HT}_{3}$ receptor antagonists.

To know about dose-responsiveness is important for two reasons. First, when larger doses are not much more efficacious than smaller doses, the smaller doses are likely to be more cost-effective. Second, smaller doses may be 
Table 3: Efficacy data

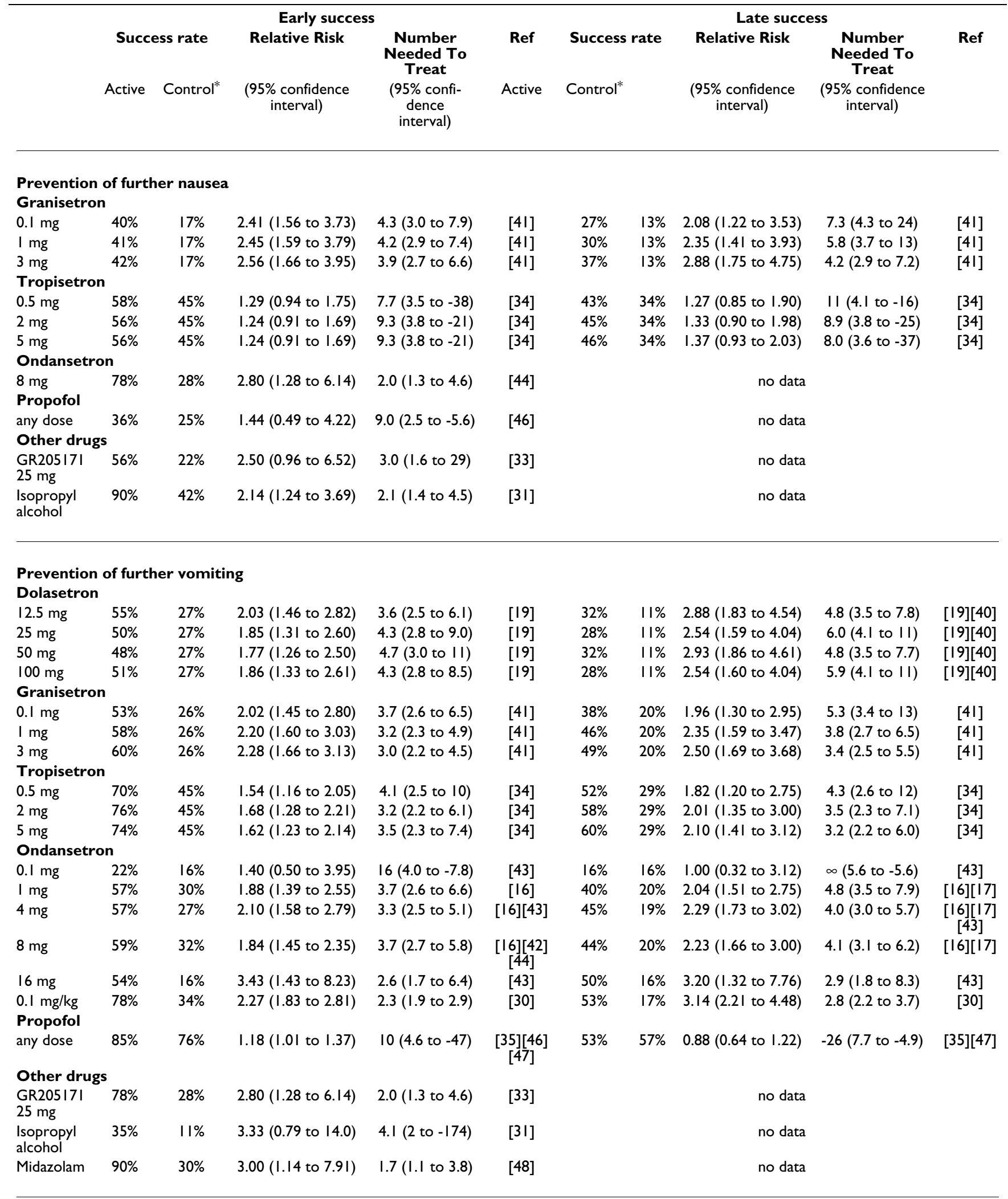

${ }^{*}$ control $=$ placebo, except in propofol trials where control $=$ intralipid; $\infty=$ infinity $($ absolute risk reduction $=0$ ) 
Table 4: Risk of headache with $5-\mathrm{HT}_{3}$ receptor antagonists

\begin{tabular}{|c|c|c|c|c|c|c|c|c|c|c|}
\hline \multirow[b]{4}{*}{ Dose } & \multirow{2}{*}{\multicolumn{3}{|c|}{$\begin{array}{c}5-\mathrm{HT}_{3} \text { receptor antagonist } \\
(\mathrm{mg})\end{array}$}} & \multicolumn{6}{|c|}{ Number with } & \multirow{4}{*}{ References } \\
\hline & & & & & Event rat & & & & Relative risk & \\
\hline & \multirow[b]{2}{*}{$\begin{array}{l}\text { Dolas- } \\
\text { etron }\end{array}$} & \multirow[b]{2}{*}{$\begin{array}{l}\text { Grani- } \\
\text { setron }\end{array}$} & \multirow[b]{2}{*}{$\begin{array}{l}\text { Tropi- } \\
\text { setron }\end{array}$} & \multirow[b]{2}{*}{$\begin{array}{l}\text { Ondans } \\
\text { etron }\end{array}$} & \multirow[b]{2}{*}{ Active } & \multirow[b]{2}{*}{ Placebo } & \multicolumn{2}{|c|}{ headache/Total number } & \multirow[b]{2}{*}{$\begin{array}{l}95 \% \text { confidence } \\
\text { interval }\end{array}$} & \\
\hline & & & & & & & Active & Placebo & & \\
\hline Low: & 12.5 & 0.1 & 0.5 & I & $7.7 \%$ & $10.4 \%$ & $4|/ 53|$ & $55 / 531$ & $0.75(0.5 \mathrm{I}$ to I.I0) & $\begin{array}{l}{[16][19][34][40]} \\
{[41]}\end{array}$ \\
\hline Medium: & 25,50 & 1 & 2 & 4 & $9.3 \%$ & $9.3 \%$ & $71 / 765$ & $55 / 591$ & $\mathrm{I} .09$ (0.78 to I.52) & $\begin{array}{l}{[16][19][34][40]} \\
{[4 I][45]}\end{array}$ \\
\hline High: & 100 & 3 & 5 & 8 & $13.3 \%$ & $9.9 \%$ & $74 / 556$ & $56 / 567$ & $1.36(0.98$ to I.88) & $\begin{array}{l}{[16][19][34][40]} \\
{[41][42]}\end{array}$ \\
\hline
\end{tabular}

Doses have been grouped arbitrarily into "low", "medium", and "high"; these groups do not represent equipotent doses.

less harmful. As in previous analyses, we have chosen a combined approach to test for dose-responsiveness, taking into account graphical display, an estimate of the statistical significance of the difference between doses, and an estimate of the clinical relevance of such a difference. Our aim was to facilitate decision-making using a pragmatic and robust method which is clinically applicable. Thus, the major question whether or not to increase the dose of an antiemetic was mainly based on the clinical relevance of any improvement in efficacy. Therefore, and since these trials represented clinically homogenous data and very similar control event rates, we were using the absolute risk reduction and the number-needed-to-treat, respectively, to test for dose-responsiveness. For none of the setrons there was strong evidence of dose-responsiveness. For ondansetron there was some weak evidence; however, the subthreshold dose $(0.1 \mathrm{mg})$ and the dose that was 160 times higher $(16 \mathrm{mg})$ were tested in a very limited number of patients only.

The lack of a clear dose-response with these $5-\mathrm{HT}_{3}$ receptor antagonists in the surgical setting is surprising for two reasons. First, wide dose ranges were tested. For dolasetron, the doses differed by a factor of 8 , for tropisetron by 10 , and for granisetron even by 30 . This means that for granisetron, for instance, 30 times more drug costs must be spent to safe an additional 10\% of vomiting patients from further vomiting (Table 3). Second, for ondansetron there is evidence from systematic review that the optimal dose to prevent PONV is likely to be $8 \mathrm{mg}$ [13]. For therapeutic purposes, however, $1 \mathrm{mg}$ seems to be as efficacious as higher doses (Table 3, Figure 2). This is interesting both from a pharmacologic and an economic point of view. Pharmacologically, these data suggest that minimal amounts of ondansetron are needed to block $5-\mathrm{HT}_{3}$ receptors in a vomiting patient, but that much higher doses are needed to block these receptors prophylactically. We do not know if this is a kinetic or a dynamic phenomenon, but we may assume that it applies to all setrons. Economically, this observation is interesting since these drugs are relatively expensive. It may make a difference if all surgical patients (including those who actually will not need an antiemetic) receive prophylactically a high dose of an expensive drug, or if only those who are suffering from PONV symptoms will be treated with a small (but effective) dose of the same drug. The pragmatic clinical message is that with all these tested $5-\mathrm{HT}_{3}$ receptor antagonists, minimal doses may be used to treat established PONV symptoms, while much higher doses are needed to try to prevent these symptoms. Obviously, with the treatment strategy, a patient will have to vomit first or suffer some degree of nausea before she gets a rescue medication.

Headache is a well known adverse effect of $5-\mathrm{HT}_{3}$ receptor antagonists [13,50]. With the highest doses of all four $5-\mathrm{HT}_{3}$ receptor antagonists, the risk of headache compared with placebo was increased (although not statistically significant). This may be regarded as a further argument to use minimal effective doses of these drugs. None of the other well known adverse effects of $5-\mathrm{HT}_{3}$ receptor antagonists, elevated liver enzymes or constipation [13,50], have been reported in more than one trial.

A final issue relates to the lack of valid data on the therapeutic efficacy of the classic antiemetics in the postoperative period. This is problematic since many of these old antiemetics have been used for decades, and they are still widely used in daily clinical practice. However, unless their relative efficacy is established, there cannot be evidence-based treatment recommendations for PONV. There is a plethora of randomised clinical trials on the prevention of PONV symptoms and a paucity of therapeutic trials, and there may be several reasons for this 
discrepancy. Therapeutic trials are logistically more difficult to perform. If the baseline risk of 30\% vomiting and nauseous postoperative patients is about right, then of 1,000 patients who have given their informed consent to take part in a therapeutic trial, 300 may eventually suffer from PONV symptoms. Only these may then be randomised and treated, and need to be followed-up for 24 hours. Trialists may prefer to give a drug to all patients prophylactically, and then see what's happening. Also, manufacturers may have more commercial interest in prophylaxis strategies than in treatment strategies, since then all patients will receive the drug and not only those who actually need it. Most valid data came from large multicentre trials that have been designed and sponsored by the manufacturers of the modern $5-\mathrm{HT}_{3}$ receptor antagonists. Most of these trials were laudable examples of placebo-controlled dose-finding studies. However, none of them compared two $5-\mathrm{HT}_{3}$ receptor antagonists. And one only compared a $5-\mathrm{HT}_{3}$ receptor antagonist with a classic antiemetic [36]. In that trial, ondansetron $4 \mathrm{mg}$ was superior to metoclopramide 10 $\mathrm{mg}$. Metoclopramide $10 \mathrm{mg}$, however, is not antiemetic [51].

It seems that manufacturers of old classic antiemetics are not interested in testing their compounds in well designed large trials; these drugs are widely used anyway despite the lack of evidence-based high-quality data. For none of these classic antiemetics, a dose-response has been established, and for none the optimal dose to treat established PONV is known. Manufacturers of the new compounds have produced high-quality data. However, they are not keen to compare their drugs with other new active comparators, or with effective regimens of old antiemetics. Also, in these trials, anti-nausea data are often underreported. This may lead to a false impression of antiemetic efficacy.

\section{Conclusions}

In postoperative patients who are suffering from nausea or vomiting, $5-\mathrm{HT}_{3}$ receptor antagonist have some effect on vomiting and less so on nausea. Minimal effective doses should be used since these are as effective as much higher doses. There is a lack of valid data for the classic antiemetics. Evidence-based treatment strategies that take into account all possible antiemetic interventions have not yet been established [49]. If anaesthesiologists do not want to rely exclusively on drugs that are expensive and of limited anti-nausea efficacy they need to design valid trials with older classic antiemetics. Future trials should be randomised, properly double-blind, and placebo-controlled. They should report on both short (up to 6 hours after treatment) and long (to 24 hours) observation periods, although it would be useful to also report on the delay until the antiemetic treatment shows effica- cy. Nausea and vomiting should be separately reported, and adverse drug reactions need to be documented.

\section{Funding}

Prosper Grant $\mathrm{N}^{\circ}$ 3233-051939.97 from the Swiss National Research Foundation (MRT).

\section{Competing Interests}

None declared.

\section{Acknowledgements}

We thank Eli Alon, MD, who responded to our enquiry. We thank Daniel Haake from the Medical libraries of the Centre Medical Universitaire, Geneva University, Geneva, for his help in searching electronic databases.

\section{References}

I. Clergue F, Auroy Y, Pequignot F, Jougla E, Lienhart A, Laxenaire MC: French survey of anesthesia in 1996. Anesthesiology 1999, 9I:I509-1520

2. Tramèr MR: Part I. A rational approach to the control of postoperative nausea and vomiting: evidence from systematic reviews. Part I. Efficacy and harm of antiemetic interventions, and methodological issues. Acta Anaesthesiol Scand 2001, 45:4-13

3. Henzi I, Walder B, Tramèr MR: Dexamethasone for the prevention of postoperative nausea and vomiting: a quantitative systematic review. Anesth Analg 2000, 90:186-194

4. Tramèr MR, Reynolds DJM, Moore RA, McQuay HJ: When placebo controlled trials are essential and equivalence trials are inadequate. BMJ I998, 3 I 7:780-785

5. Fortney JT, Gan TJ, Graczyk S, Wetchler B, Melson T, Khalil S: A comparison of the efficacy, safety, and patient satisfaction of ondansetron versus droperidol as antiemetics for elective outpatient surgical procedures. S3A-409 and S3A-4 I 0 Study Groups. Anesth Analg 1998, 86:731-738

6. Tramèr MR, Phillips C, Reynolds DJM, Moore RA, McQuay HJ: Costeffectiveness of ondansetron for postoperative nausea and vomiting. Anaesthesia 1999, 54:226-235

7. Jadad AR, Moore RA, Carroll D, Jenkinson C, Reynolds DJM, Gavaghan $\mathrm{DJ}$, McQuay $\mathrm{HJ}$ : Assessing the quality of reports of randomized clinical trials: is blinding necessary? Control Clin Trials 1996, I 7:1-12

8. Tramèr MR, Moore RA, Reynolds DJM, McQuay HJ: A quantitative systematic review of ondansetron in treatment of established postoperative nausea and vomiting. BMJ I997, 3 | 4: 1088-1092

9. Morris JA, Gardner MJ: Calculating confidence intervals for relative risk, odds ratio, and standardised ratios and rates. Statistics with confidence - Confidence intervals and statistical guidelines. Ed: D.G. Altman and M.J. Gardner. BMJ Books, London, 199550-63

10. Gavaghan DJ, Moore RA, McQuay HJ: An evaluation of homogeneity tests in meta-analysis in pain using simulations of individual patient data. Pain 2000, 85:4I5-424

II. Laupacis A, Sackett DL, Roberts RS: An assessment of clinically useful measures of the consequences of treatment. $N$ Engl Med | 988, 3 | 8: |728-33

12. Cook RJ, Sackett DL: The number needed to treat: a clinically useful measure of treatment effect. BMJ I 995, 3 I 0:452-454

13. Tramèr MR, Reynolds DJM, Moore RA, McQuay HJ: Efficacy, doseresponse, and safety of ondansetron in prevention of postoperative nausea and vomiting: A quantitative systematic review of randomized placebo-controlled trials. Anesthesiology 1997, 87:I277-1289

14. Altman D: Confidence intervals for the number needed to treat. BM] 1998, 3 1 7:1309-1312

15. Tramèr M, Moore A, McQuay H: Meta-analytic comparison of prophylactic antiemetic efficacy for postoperative nausea and vomiting: propofol anaesthesia vs omitting nitrous oxide vs a total i.v. anaesthesia with propofol. Br J Anaesth 1997, 78:256-259 
16. Du Pen S, Scuderi P, Wetchler B, Sung YF, Mingus M, Claybon L, Leslie J, Talke P, Apfelbaum J, Sharifi Azad S, et al: Ondansetron in the treatment of postoperative nausea and vomiting in ambulatory outpatients: a dose-comparative, stratified, multicentre study. Eur J Anaesthesiol 1992, 9 Suppl 6:55-62

17. Claybon L: Single dose intravenous ondansetron for the 24hour treatment of postoperative nausea and vomiting. Anaesthesia 1994, 49 Suppl:24-29

18. Scuderi P, Wetchler B, Sung YF, Mingus M, Du Pen S, Claybon L, Leslie J, Talke P, Apfelbaum J, Sharifi-Azad S, et al: Treatment of postoperative nausea and vomiting after outpatient surgery with the 5-HT3 antagonist ondansetron. Anesthesiology 1993, 78:1520

19. Kovac AL, Scuderi PE, Boerner TF, Chelly JE, Goldberg ME, Hantler $\mathrm{CB}$, Hahne WF, Brown RA: Treatment of postoperative nausea and vomiting with single intravenous doses of dolasetron mesylate: A multicenter trial. Anesth Analg 1997, 85:546-552

20. Kovac A, Mingus M, Sung YF, Neary M: Reduced resource utilization in patients treated for postoperative nausea and vomiting with dolasetron mesylate. J Clin Anesth 1999, I I:235-24 I

21. Collini S, Di Donato A, Brauneis S, Turkiewicz AM, Zaccheao AR, Pinto G: Il vomito postoperatorio in oftalmochirurgia: profilassi, tattamento e ritrattamento in uno studio randomizzato in doppio cieco con ondansetron. Acta Anaesth Ital 1995, 46:49-56

22. Tate Sylvina MB: Peppermint oil: a treatment for postoperative nausea. J Adv Nursing 1997, 26:543-549

23. Kauste A, Tuominen M, Heikkinen H, Gordin A, Korttila K: Droperidol, alizapride and metoclopramide in the prevention and treatment of postoperative emetic sequelae. Eur J Anaesth 1986, 3:1-9

24. Korttila K, Kauste A, Auvinen J: Comparison of domperidone, droperidol, and metoclopramide in the prevention and treatment of nausea and vomiting after balanced general anesthesia. Anesth Analg 1979, 58:396-400

25. Scuderi PE, James RL, Harris L, Mims GR3: Antiemetic prophylaxis does not improve outcomes after outpatient surgery when compared to symptomatic treatment. Anesthesiology 1999, 90:360-37I

26. Roy $\mathrm{P}$, Patel $\mathrm{NH}$, Miller AJ: A comparison of controlled release metoclopramide and domperidon in the treatment of nausea and vomiting. BJCP I99I, 45:247-25I

27. Van Leeuwen L, Helmers JH: The efficacy of domperidone ( $R$ $338 I 2)$ in the treatment of postoperative vomiting. Anaesthesist 1980, 29:490-3

28. Heim C, Münzer T, Listyo R: Ondansetron versus Droperidol. Postoperativer therapeutischer Einsatz bei Nausea und Erbrechen. Vergleich von Wirkung, Nebenwirkungen und Akzeptanz bei gynäkologischen, stationären Patientinnen. Anaesthesist 1994, 43:504-509

29. Ummenhofer W, Frei FJ, Urwyler A, Kern C, Drewe J: Effects of ondansetron in the prevention of postoperative nausea and vomiting in children. Anesthesiology 1994, $81: 804-810$

30. Khalil S, Rodarte A, Weldon BC, Weinstein M, Grunwald Z, Ginsberg B, Kaye R, Otto A, Wheeler M, Lawhorn CD, et al: Intravenous ondansetron in established postoperative emesis in children. Anesthesiology 1996, 85:270-6

31. Wang SM, Hofstadter MB, Kain ZN: An alternative method to alleviate postoperative nausea and vomiting in children. / Clin Anesth 1999, II:23I-234

32. Lacroix G, Lessard MR, Trepanier CA: Treatment of post-operative nausea and vomiting: Comparison of propofol, droperidol and metoclopramide. Can J Anaesth 1996, 43:I I5-I 20

33. Diemunsch P, Schoeffler P, Bryssine B, Cheli-Muller LE, Lees J, McQuade BA, Spraggs CF: Antiemetic activity of the NKI receptor antagonist GR205 I7I in the treatment of established postoperative nausea and vomiting after major gynaecological surgery. Br JAnesth 1999, 82:274-276

34. Alon E, Buchser E, Herrera E, Christiaens F, De Pauw C, Ritter L, Hulstaert F, Grimaudo V: Tropisetron for treating established postoperative nausea and vomiting: a randomized, doubleblind, placebo-controlled study. Anesth Analg 1998, 86:617-623

35. Gan TJ, El-Molem H, Ray J, Glass PSA: Patient-controlled antiemesis. A randomized, double-blind comparison of two doses of propofol versus placebo. Anesthesiology 1999, 90:1564-70

36. Diemunsch P, Conseiller C, Clyti N, Mamet JP: Ondansetron compared with metoclopramide in the treatment of established postoperative nausea and vomiting. Br J Anaesth 1997, 79:322326

37. Fujii $Y$, Tanaka $\mathrm{H}$, Kawasaki $\mathrm{T}$ : Randomized clinical trial of granisetron, droperidol and metoclopramide for the treatment of nausea and vomiting after laparoscopic cholecystectomy. $\mathrm{Br}$ J Surg 2000, 87:285-288

38. Fujii $Y$, Tanaka $H$, Somekawa $Y$ : Granisetron, droperidol, and metoclopramide for the treatment of established postoperative nausea and vomiting in women undergoing gynecologic surgery. Am J Obstet Gynecol 2000, I 82:13-16

39. Stienstra R, Samhan YM, el Mofty M, de Bont LE, Bovill JG: Doubleblind comparison of alizapride, droperidol and ondansetron in the treatment of post-operative nausea. Eur J Anaesthesiol 1997, 1 4:290-294

40. Diemunsch P, Leeser J, D'Hollander A, Bradburn BG, Paxton D, Whitmore J, Panouillot P, Navé S, Brown RA, Hahne WF: Intravenous dolasetron mesilate ameliorates postoperative nausea and vomiting. Can J Anaesth 1997, 44:173-18|

4I. Taylor AM, Rosen M, Diemunsch PA, Thorin D, Houweling PL: A double-blind, parallel-group, placebo-controlled, dose-ranging, multicenter study of intravenous granisetron in the treatment of postoperative nausea and vomiting in patients undergoing surgery with general anesthesia. J Clin Anesth 1997, 9:658-663

42. Bodner M, White PF: Antiemetic efficacy of ondansetron after outpatient laparoscopy. Anesth Analg 199I, 73:250-254

43. Rung GW, Claybon L, Hord A, Patel C, Kallgren M, Koppel J, Benedetti C, Creed M, Asgharian A, Bryson: Intravenous ondansetron for postsurgical opioid-induced nausea and vomiting. Anest Analg 1997, 84:832-838

44. GE Larijani, I Gratz, M Afshar, S Minassian: Treatment of postoperative nausea and vomiting with ondansetron: a randomized, double-blind comparison with placebo. Anesth Analg I99|, 73:246-9

45. Polati E, Verlato G, Finco G, Mosaner W, Grosso S, Gottin L, Pinaroli AM, Ischia S: Ondansetron versus metoclopramide in the treatment of postoperative nausea and vomiting. Anesth Analg 1997, 85:395-399

46. Harper I, Della Marta E, Owen H, Plummer J, Ilsley A: Lack of efficacy of propofol in the treatment of early postoperative nausea and vomiting. Anaesth Intensive Care 1998, 26:366-370

47. Zestos MM, Carr AS, McAuliffe G, Smith HS, Sikich N, Lerman J: Subhypnotic propofol dose not treat postoperative vomiting in children after adenotonsillectomy. Can J Anaesth 1997, 44:40I-404

48. Di Florio T, Goucke CR: The effect of midazolam on persistent postoperative nausea and vomiting. Anaesth Intensive Care 1999, 27:38-40

49. Tramèr MR: Part II. A rational approach to the control of postoperative nausea and vomiting. Evidence from systematic reviews. Part II. Recommendations for prevention and treatment, and research agenda. Acta Anaesthesiol Scand $200114-19$

50. Tramèr MR, Reynolds DJM, Stoner NS, Moore RA, McQuay HJ: Efficacy of 5-HT3 receptor antagonists in radiotherapy-induced nausea and vomiting: a quantitative systematic review. Eur J Cancer 1998, 34:1836-44

51. Henzi I, Walder B, Tramèr MR: Metoclopramide in the prevention of postoperative nausea and vomiting - a quantitative systematic review of randomised placebo-controlled studies. Br J Anaesth |999, 83:76|-77| 